\title{
Preprints as a complement to the journal system in biology
}

\author{
Jessica Polka \\ ASAPbio, California, USA \\ E-mail: Jessica.polka@asapbio.org
}

\begin{abstract}
ASAPbio (Accelerating Science and Publication in biology) is a group of biologists at different stages of their career with an interest of promoting and accelerating knowledge sharing in biology. Preprint publishing in biology is taking off, and this article outlines the fundamental compatibility of preprints with the journal system, as well as the specific contribution of preprints to solving several important problems in publishing and fostering innovation.
\end{abstract}

Keywords: Scholarly journals, preprint, peer review, biology

Publishing in biology isn't what it used to be. Hyper-competition among researchers drives us to squeeze as much impact from every publication as is possible. It takes $\mathrm{PhD}$ students longer to graduate, it takes them longer to publish their first article, and publications today include more data than they did 30 years ago [3]. Hence, new and relevant knowledge is not made available as quickly as it could or should be.

Some venues encourage faster publication, but these are not always 'impactful' venues. An interesting alternative is the 'preprint', a manuscript posted online before journal-organized peer review.

\section{Preprints and journals are compatible}

The key element making preprints attractive to biologists is their high compatibility with the existing journal system. While the scientist is waiting for editorial curation, evaluation, and peer review, the manuscript is online already, where it can be viewed by anyone (see Fig. 1).

In physics, preprints have been used for more than 25 years, and more than one million manuscripts are available on arXiv.org. Quantitative biology has been present on arXiv for more than a decade, and bioRxiv launched in 2013. In the past five years, the number of preprints in biology has taken off (Fig. 2). Notably, the cost of publishing preprints can be very low at an average of $\$ 10$ per preprint deposited in arXiv. $^{1}$

\section{Preprints as problem-solver}

Biologists have discovered that preprints solve several key problems in the areas of access to literature, peer recognition, and career progression (see Table 1).

\footnotetext{
${ }^{1}$ https://confluence.cornell.edu/display/arxivpub/arXiv+Budgets+and+Reserve+Fund+Policy.
} 


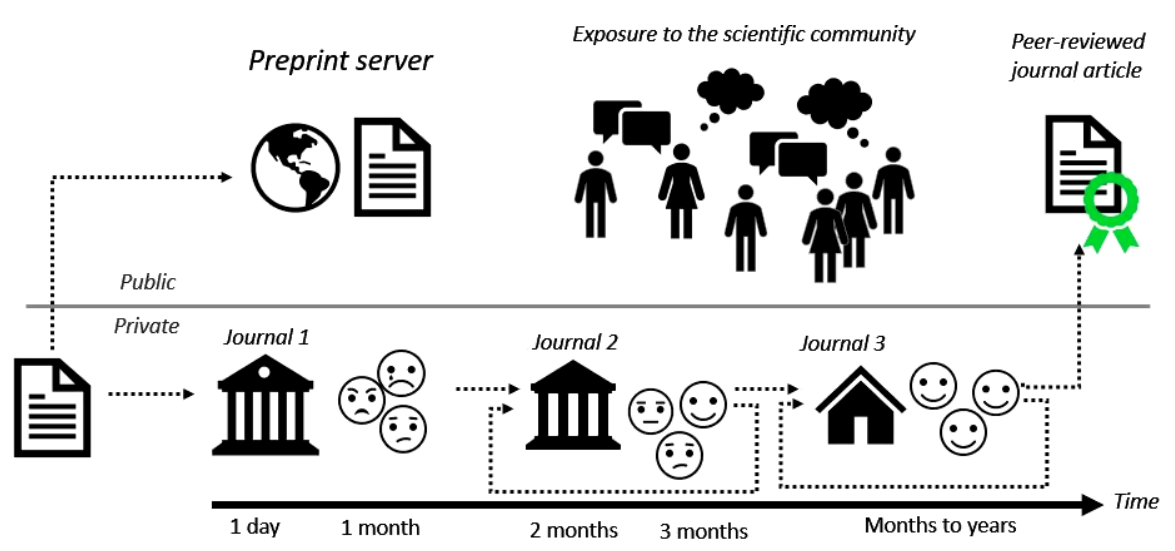

Fig. 1. Preprints and journals.

While preprints are an effective solution in these areas, some concerns remain. Some say that we can't be trusted to share our work before peer review because too many papers of poor quality will be made available. However, researchers are very concerned about reputations, and we carefully vet material made public before peer review, like conference talks or posters. Another concern is that journals will not accept the submission of papers available as preprints. However, journal policies now generally allow for preprints in the basic life sciences. ${ }^{2}$ Moreover, the International Committee of Medical Journal Editors has clarified that a preprint is not a competing publication and that the 'Inglefinger rule' against dual publication thus does not apply. ${ }^{3}$ More than 3000 medical journals endorse the ICMJE rec-

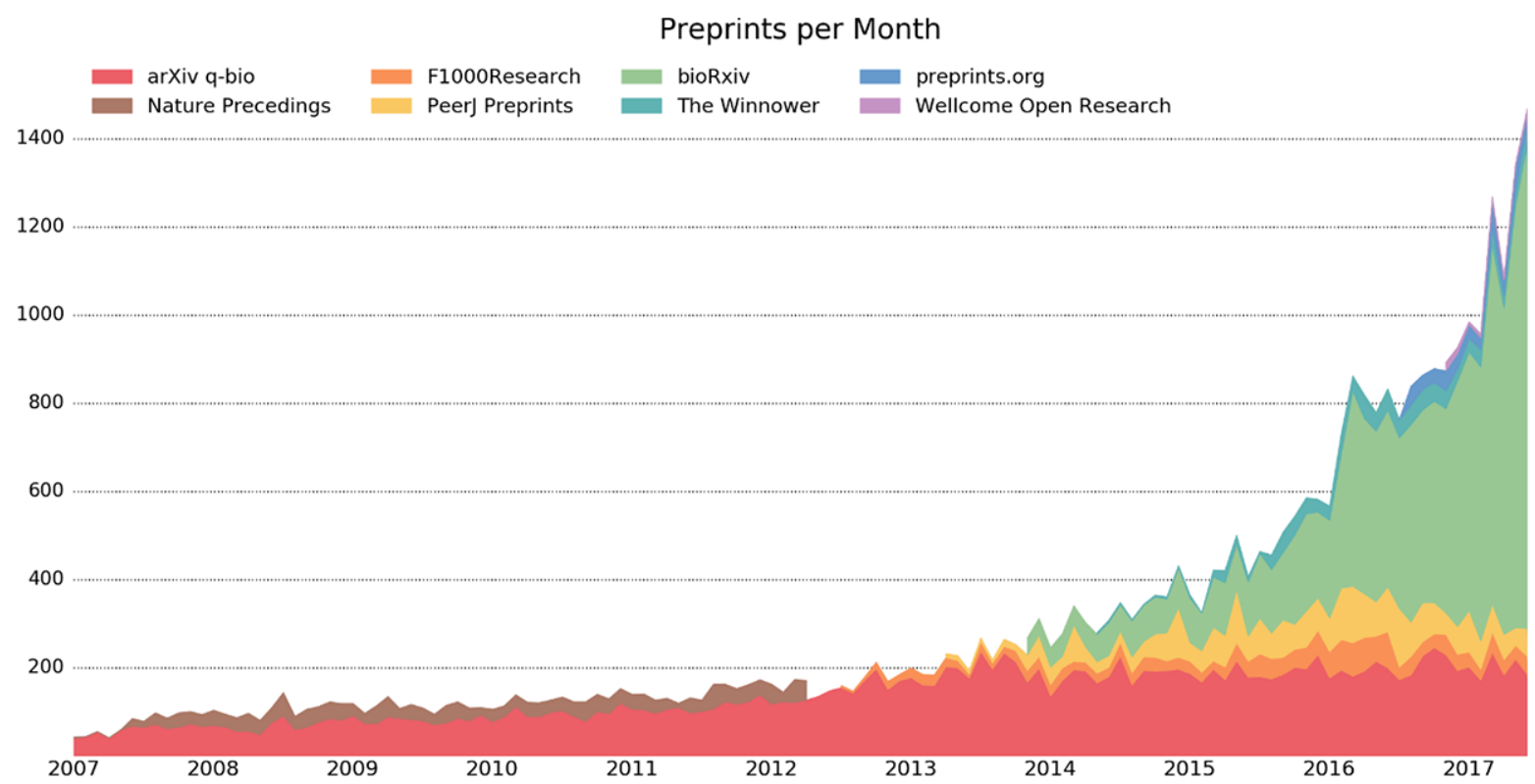

Fig. 2. Preprints across disciplines (https://github.com/OmnesRes/prepub/blob/master/analyses/june_preprints.png - graph by Jordan Anaya of PrePubMed (License: https://github.com/OmnesRes/prepub/blob/master/LICENSE)).

\footnotetext{
${ }^{2} \mathrm{https}: / /$ en.wikipedia.org/wiki/List_of_academic_journals_by_preprint_policy.

${ }^{3} \mathrm{http}: / /$ www.icmje.org/recommendations/.
} 
Table 1

Key problems and the solution preprints provide

\begin{tabular}{|c|c|}
\hline Problem & Solution \\
\hline Lack of access to literature & $\begin{array}{l}\text { Preprints are immediately available to everyone around } \\
\text { the world }\end{array}$ \\
\hline $\begin{array}{l}\text { Students and postdocs stay in training programs longer to } \\
\text { publish }\end{array}$ & Preprints can be evaluated for a thesis and job application \\
\hline Your recent work is invisible to grant and award committees & Preprints make you most recent work visible \\
\hline Your colleagues can't see your recent work & Preprints stimulate collaborations \\
\hline Peer review is based on a small number of opinions & Preprints allow the whole world to provide feedback \\
\hline $\begin{array}{l}\text { Lack of transparency in the review process creates confusion } \\
\text { about priority of discovery }\end{array}$ & $\begin{array}{l}\text { Preprints are permanent and time-stamped - evidence of } \\
\text { what work was done when }\end{array}$ \\
\hline Laboratories are keeping knowledge secret & $\begin{array}{l}\text { Preprints are immediately accessible, allowing research } \\
\text { to advance overall }\end{array}$ \\
\hline
\end{tabular}

ommendations, including the New England Journal of Medicine (of which Inglefinger was the editor). ${ }^{4}$ Others worry about how we can ensure that the disclosure of data is ethical. Many preprint servers have instituted a system of screening manuscripts prior to posting to help avoid such problems.

Perhaps the most important concern is whether the posting of a preprint may mean a risk of getting 'scooped'. After all, a preprint is not a publication. However, preprint submissions are date-stamped. In physics, arXiv postings have been accepted as priority claims. Moreover, preprints are citable, hence proper attribution must be expected.

\section{Posting preprints is a good experience}

ASAPbio (Accelerating Science and Publication in biology) is a group of biologists at different stages of their career with an interest of promoting and accelerating knowledge sharing in biology. Beginning in 2015 and led by our founder Ron Vale, we began discussing whether preprints could play a larger role in the publication system in biology. To assess this, we conducted an informal survey and found that biologists posting preprints overwhelmingly report a positive experience (see Fig. 3, with 392 total responses). ${ }^{5}$

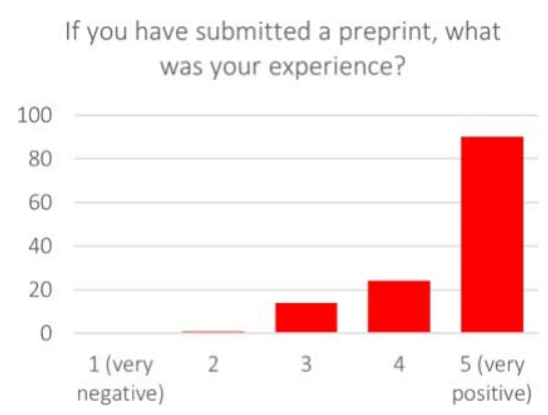

Fig. 3. Scholarly experience with preprints.

\footnotetext{
${ }^{4}$ http://www.icmje.org/journals-following-the-icmje-recommendations/.

${ }^{5} \mathrm{http}$ ://asapbio.org/survey.
} 
In February 2016, the group organized a meeting at HHMI to bring together the relevant stakeholders, including funders, journals, and promotion committees. ${ }^{6}$ Attendees of this meeting were extremely positive about the prospect of an expanded role for preprints, as described in the meeting report [1].

We are currently looking at the culture and incentives in biology to promote the deposit of preprints. Several funders have made explicit their endorsement of preprints, among them the Human Frontiers Science Program, Helmsley Trust, and Simons Foundation in the United States; and the Wellcome Trust and Medical Research Council in the United Kingdom.

\section{Journals innovating with preprints}

What benefits can we reap when journals take on innovating with preprints? Four examples stand out:

1. The direct transfer of the preprint from bioRxiv to the journal of choice for review and publication. ${ }^{7}$

2. The emergence of a new kind of marketplace, by which journals pro-actively scan preprints and invite submission, as exemplified by PLOS Genetics" "Preprint Editors." 8

3. The formal extension of the scoop protection to the posting of the preprint, as pioneered by $E M B O$ Journal. ${ }^{9}$

4. Linking preprints to the final journal version of record, as done by Biophysical Journal [2].

\section{References}

[1] J.M. Berg et al., Preprints for the life sciences, Science 352(6288) (2016), 899-901. doi:10.1126/science.aaf9133.

[2] L.M. Loew et al., Ushers in new editorial board members and more, Biophysical Journal 112(1) (2017), E01-E02. doi:10. 1016/j.bpj.2016.12.014.

[3] R.D. Vale, Accelerating scientific publication in biology, PNAS 112(44) (2015), 13439-13446. doi:10.1073/pnas. 1511912112.

\footnotetext{
${ }^{6}$ http://asapbio.org/about-2.

${ }^{7} \mathrm{http}: / /$ www.biorxiv.org/about-biorxiv

${ }^{8} \mathrm{http} / / /$ journals.plos.org/plosgenetics/s/editorial-board\#loc-preprint-editors.

${ }^{9} \mathrm{http} / / / \mathrm{embo.org} /$ news/articles/2017/protected-as-soon-as-you-unveil-your-masterpiece.
} 